Pacific Journal of Mathematics

INVARIANT SUBSPACES AND UNSTARRED OPERATOR 


\title{
INVARIANT SUBSPACES AND UNSTARRED OPERATOR ALGEBRAS
}

\author{
D. SARASON
}

It is proved in the present paper that if $A$ is a normal Hilbert space operator, and if the operator $B$ leaves invariant every invariant subspace of $A$, then $B$ belongs to the weakly closed algebra generated by $A$ and the identity. This may be regarded as a refinement of the von Neumann double commutant theorem. A generalization is given in which the single operator $A$ is replaced by a commuting family of normal operators. Also the same result is proved for the case where $A$ is an analytic Toeplitz operator.

The results to be obtained will now be described in greater detail. Theorem 1 refines the following well-known result.

THEOREM 0. If $A$ is a normal operator on a Hilbert space $H$, and if the operator $B$ on $H$ commutes with every projection that commutes with $A$, then $B$ belongs to the weakly closed star-algebra generated by $A$ and the identity.

This is essentially the von Neumann double commutant theorem; see $[13$, p. 64] for the separable case and [11, p. 173] for the nonseparable case, both in conjunction with [3, p. 43, Lemma 6].

To say that an operator $B$ commutes with every projection that commutes with the operator $A$ amounts to saying that $B$ is reduced by every subspace that reduces $A$. The following theorem thus has a stronger hypothesis than Theorem 0 and draws a stronger conclusion.

THEOREM 1. If $A$ is a normal operator on a Hilbert space $H$, and if the operator $B$ on $H$ leaves invariant every invariant subspace of $A$, then $B$ belongs to the weakly closed algebra generated by $A$ and the identity.

Theorem 1 can be obtained very easily from Theorem 0 . A proof is presented in $\S 2$. As an immediate consequence of Theorem 1 we have the

CoRollary. If $A$ is a normal Hilbert space operator, then the weakly closed algebra generated by $A$ is a star-algebra if and only if every invariant subspace of $A$ is a reducing subspace.

Received January 7, 1965. 
For the case where $A$ is unitary this is closely related to a theorem of R. W. Goodman [5]. We might mention that Wermer [14] has given a simple example of a normal operator $A$ which has no nonreducing invariant subspaces but is such that $A^{*}$ is not the weak limit of any sequence of polynomials in $A$.

Theorems 0 and 1 can both be regarded as special cases of a more general result.

THEOREM 2. If $\mathscr{A}$ is a commutative, identity containing, weakly closed algebra of normal operators on a Hilbert space $H$, and if the operator $B$ on $H$ leaves invariant every invariant subspace of $\mathscr{A}$, then $B$ belongs to $\mathscr{A}$.

If $\mathscr{A}$ is a star-algebra this is again essentially the von Neumann double commutant theorem, a well-known result. The theorem for the case where $A$ is not a star-algebra can be obtained from the case where it is by the same reasoning that yields Theorem 1 from Theorem 0 . A proof is briefly indicated in $\$ 2$.

Theorem 1 is not true in general for nonnormal operators; one can give a trivial counter-example involving two-by-two matrices. However there is a class of nonnormal operators for which Theorem 1 does hold, namely the analytic Toeplitz operators (to be defined later).

Theorem 3. If $A$ and $B$ are analytic Toeplitz operators, and if $B$ leaves invariant every invariant subspace of $A$, then $B$ belongs to the weakly closed algebra generated by $A$ and the identity.

The analytic Toeplitz operators form an algebra; in fact they form the weakly closed algebra generated by the so-called unilateral shift operator and the identity. The special properties of the shift will enable us to prove Theorem 3 in the same way as Theorem 1. This is done in $\S 3$.

One question that suggests itself is: which operators besides the shift generate the algebra of analytic Toeplitz operators? In view of Theorem 3, an equivalent question is: which analytic Toeplitz operators have precisely the same invariant subspaces as the shift? This problem is investigated in detail in the following paper. In $\S 4$ of the present paper a few immediate conclusions are obtained.

2. Proof of Theorem 1. The reader is assumed familiar with the basic theory of normal operators, and we shall employ elementary results from this theory without further explanation. In terminology we follow Halmos's book [6]. First some notations are needed. Suppose that $A$ is a normal operator on a Hilbert space $H$, and let $E$ be 
the spectral measure of $A$. (We regard $E$ as defined on the Borel subsets of the complex plane.) For $x$ in $H$ we let $E_{x}$ denote the Borel measure on the plane defined by $E_{x}(S)=(E(S) x, x)$. For $m$ a natural number we let $H_{m}$ denote the direct sum of $H$ with itself $m$ times, $A_{m}$ the direct sum of $A$ with itself $m$ times (regarded as an operator on $H_{m}$ ), and $E_{m}$ the direct sum of $E$ with itself $m$ times (so $E_{m}$ is the spectral measure of $A_{m}$ ).

The following lemma is the essential step in the proof of Theorem 1.

Lemma 1. With the above notations, let $B$ be an operator on $H$ which leaves invariant every invariant subspace of $A$. Then $B_{m}$ leaves invariant every invariant subspace of $A_{m}, m=1,2,3, \cdots$.

Proof. It will be enough to show that every cyclic invariant subspace of $A_{m}$ is invariant under $B_{m}$. To this end, let $x$ be a vector in $H_{m}$ and let $M$ be the smallest reducing subspace of $A_{m}$ containing $x$. The measure $\left(E_{m}\right)_{x}$ is absolutely continuous with respect to $E$, and so there is a vector $y$ in $H$ such that $E_{y}=\left(E_{m}\right)_{x}$. Let $N$ be the smallest reducing subspace of $A$ containing $y$. Since $\left(E_{m}\right)_{x}=E_{y}$, the operators $A_{m} \mid M$ and $A \mid N$ are unitarily equivalent. Hence there is an isometry $V$ of $N$ onto $M$ such that $A_{m} \mid M=V A V^{-1}$. It follows that if $q$ is any complex polynomial in two variables, then $q\left(A_{m}, A_{m}^{*}\right) \mid M=V q\left(A, A^{*}\right) V^{-1}$. But by Theorem 0 , there is a net $\left\{q_{i}\right\}$ of such polynomials with $q_{i}\left(A, A^{*}\right) \rightarrow B$ weakly. Therefore also $q_{i}\left(A_{m}, A_{m}^{*}\right) \rightarrow B_{m}$ weakly. It follows that $B_{m} \mid M=V B V^{-1}$. Hence $V$ maps invariant subspaces of $B$ onto invariant subspaces of $B_{m}$. Let $L$ be the smallest invariant subspace of $A_{m}$ containing $x$. Then $V^{-1} L$ is invariant under $A$, and therefore also under $B$. Hence $L$ is invariant under $B_{m}$, and the proof of the lemma is complete.

Proof of Theorem 1. Let $A$ and $B$ satisfy the hypotheses of Theorem 1. Let $x_{1}, \cdots, x_{m}, y_{1}, \cdots, y_{m}$ be unit vectors in $H$, let $\varepsilon$ be a positive number, and define $\mathscr{V}$ to be the set of all operators $T$ on $H$ satisfying

$$
\left|\left(T x_{j}, y_{j}\right)-\left(B x_{j}, y_{j}\right)\right|<\varepsilon, \quad j=1, \cdots, m .
$$

Then $y$ is a weak neighborhood of $B$, and the family of all such sets $\mathscr{V}$ is a base of weak neighborhoods of $B$. Hence it will suffice to show that $\mathscr{Y}$ contains a polynomial in $A$. To do this we form the vector $x=x_{1} \oplus \cdots \oplus x_{m}$ in $H_{m}$. By Lemma $1, B_{m} x$ belongs to the invariant subspace of $A_{m}$ generated by $x$. Hence there is a polynomial $p$ such that $\left\|p\left(A_{m}\right) x-B_{m} x\right\|<\varepsilon$. This implies that $\left\|p(A) x_{j}-B x_{j}\right\|<\varepsilon$ for $j=1, \cdots, m$, and therefore 


$$
\left|\left(p(A) x_{j}, y_{j}\right)-\left(B x_{j}, y_{j}\right)\right| \leqq\left\|p(A) x_{j}-B x_{j}\right\|\left\|y_{j}\right\|<\varepsilon, \quad j=1, \cdots, m .
$$

Thus $p(A)$ belongs to $\mathscr{y}$, and the proof is complete.

We now sketch the proof of Theorem 2. Suppose $\mathscr{A}$ and $B$ satisfy the hypotheses of that theorem, and let $\mathscr{A}_{1}$ be the weakly closed star-algebra generated by $\mathscr{A}$. Then $\mathscr{A}_{1}$ is commutative by Fuglede's theorem [4]. Thus, by Theorem 2 for the case of staralgebras, the operator $B$ belongs to $\mathscr{A}_{1}$. Moreover there is a spectral measure $E$ such that $\mathscr{A}_{1}$ is the weakly closed star-algebra generated by the spectral projections $E(S)$ (see for example [10, p. 106]). It is now possible to repeat verbatim the argument used to prove Theorem 1 , but with the role of the operator $A$ taken by the algebra $\mathscr{A}$.

3. Analytic Toeplitz Operators. Let $C$ be the unit circle in the complex plane, regarded as a measure space with normalized Lebesgue measure. The spaces $L^{2}(C)$ and $L^{\infty}(C)$ will be denoted simply by $L^{2}$ and $L^{\infty}$. The functions $e_{n}(z)=z^{n}, n=0, \pm 1, \pm 2, \cdots$, form an orthonormal basis for $L^{2}$. The bilateral shift is the operator $W$ on $L^{2}$ defined by $(W f)(z)=z f(z)$, or equivalently by $W e_{n}=e_{n+1}$. For each $\varphi$ in $L^{\infty}$ we define the operator $\varphi(W)$ on $L^{2}$ by $\varphi(W) f=\varphi f$, and we denote by $L^{\infty}(W)$ the algebra of all such operators. It is well-known that $L^{\infty}(W)$ is the weakly closed star-algebra generated by $W$.

The invariant subspaces of the operator $W$ have been much studied; see [1], [8], [7]. One obvious invariant subspace is the subspace spanned by the basis vectors $e_{n}$ with $n \geqq 0$; we denote this subspace by $H^{2}$. If $\varphi$ is in $L^{\infty}$ and $H^{2}$ is invariant under $\varphi(W)$, then $\varphi$ obviously belongs to the algebra $H^{\infty}=H^{2} \cap L^{\infty}$. We denote by $H^{\infty}(W)$ the algebra of operators $\varphi(W)$ with $\varphi$ in $H^{\infty}$. It is well-known that $H^{\infty}(W)$ is the weakly closed algebra generated by $W$ and the identity [9, p. 19]. (The last conclusion can also be obtained by using Theorem 1 together with the known structure of the invariant subspaces of $W$.)

The unilateral shift is the operator $U=W \mid H^{2}$. For $\varphi$ in $H^{\infty}$ we define $\varphi(U)=\varphi(W) \mid H^{2}$, and we denote by $H^{\infty}(U)$ the algebra of all such operators $\varphi(U)$. The operators in $H^{\infty}(U)$ are called analytic Toeplitz operators. The assertion at the end of the preceding paragraph implies that every operator in $H^{\infty}(U)$ is a weak limit point of polynomials in $U$. On the other hand, it is known that $H^{\infty}(U)$ consists precisely of the operators on $H^{2}$ that commute with $U$ [2, Theorem 7], and consequently $H^{\infty}(U)$ is weakly closed. Thus $H^{\infty}(U)$ is the weakly closed algebra generated by $U$ and the identity. We also note that if the operator $B$ on $H^{2}$ leaves invariant every invariant subspace of $U$, then $B$ must belong to $H^{\infty}(U)$. This can be easily proved by using the fact that each complex number $a$ of modulus less than unity is an eigenvalue of unit multiplicity of $U^{*}$; the corre- 
sponding eigenvectors are the functions $h_{a}$ defined by $h_{a}(z)=(1-a z)^{-1}$. If $B$ leaves invariant every invariant subspace of $U$, then $B^{*}$ bears the same relation to $U^{*}$ and therefore has each $h_{a}$ as an eigenvector. Since the functions $h_{a}$ span $H^{2}$ this implies that $B^{*}$ commutes with $U^{*}$, which means that $B$ commutes with $U$ and therefore belongs to $H^{\infty}(U)$.

We can now get on with the proof of Theorem 3. For each natural number $m$ we let $H_{m}^{2}$ denote the direct sum of $H^{2}$ with itself $m$ times, and for $A$ an operator on $H^{2}$ we denote by $A_{m}$ the direct sum of $A$ with itself $m$ times.

Lemma 2. Let $A$ and $B$ be analytic Toeplitz operators, and suppose that every invariant subspace of $A$ is invariant under $B$. Then every invariant subspace of $A_{m}$ is invariant under $B_{m}, m=$ $1,2,3, \cdots$.

It is obvious that once this is proved, Theorem 3 will follow by the same reasoning we used above to obtain Theorem 1 from Lemma 1.

Proof of Lemma 2. As in the proof of Lemma 1, it will be enough to show that every cyclic invariant subspace of $A_{m}$ is invariant under $B_{m}$. Suppose that $x$ is a nonzero vector in $H_{m}^{2}$, and let $M$ be the smallest invariant subspace of $U_{m}$ containing $x$. Then as Halmos has shown [7, Theorem 2], the subspace $M$ is generated by a unit wandering vector of $U_{m}$, that is to say, there is in $M$ a unit vector $w$ such that $\left(U_{m}^{n} w, w\right)=0$ for $n>0$ and such that the vectors $U_{m}^{n} w$, $n \geqq 0$, span $M$. Hence we can define an isometry $V$ of $H^{2}$ onto $M$ by setting $V e_{n}=U_{m}^{n} w, n=0,1,2, \cdots$, and we have $U_{m} \mid M=V U V^{-1}$. Since $A$ and $B$ are weak limits of polynomials in $U$, it follows that $A_{m} \mid M=V A V^{-1}$ and $B_{m} \mid M=V B V^{-1}$. From this point the proof proceeds exactly as that of Lemma 1.

4. Generators of $H^{\infty}$. The weak topologies on $H^{\infty}(W)$ and $H^{\infty}(U)$ induce topologies on $H^{\infty}$ by virtue of the isomorphisms $\varphi \rightarrow \varphi(W)$ and $\varphi \rightarrow \varphi(U)$. The topology induced by $H^{\infty}(U)$ is obviously coarser than that induced by $H^{\infty}(W)$. It turns out that these two topologies are in fact identical and coincide with the weak-star topology of $H^{\infty}$. A proof of this can be found in [12, Proposition 11]. We shall call a function $\varphi$ in $H^{\infty}$ a generator if the polynomials in $\varphi$ are weak-star dense in $H^{\circ}$. Theorems 1 and 2 together with the preceding remark give the following result.

Proposition 1. If $\varphi$ is in $H^{\infty}$ then the following are equivalent. (i) $\varphi$ is a generator of $H^{\infty}$. 
(ii) $\varphi(W)$ has the same invariant subspaces as $W$.

(iii) $\varphi(U)$ has the same invariant subspaces as $U$.

To conclude this paper we obtain two simple necessary conditions for a function to be a generator of $H^{\infty}$. The question of the generators of $H^{\infty}$ will be discussed in detail in the following paper. We call a function $\varphi$ on $C$ univalent almost everywhere if there is a null subset $S$ of $C$ such that $\varphi$ is univalent on $C-S$.

Proposition 2. If $\varphi$ is a generator of $H^{\infty}$ then $\varphi$ is univalent almost everywhere.

Proof. If $\phi$ is not univalent almost everywhere, then it follows from multiplicity theory that $\varphi(W)$ even has reducing subspaces that are not invariant under $W$. For a more elementary proof we can argue as follows. If $\phi$ is a generator of $H^{\infty}$, then $e_{1}$ belongs to the invariant subspace of $\varphi(W)$ generated by $e_{0}$. This implies that there is a sequence of polynomials $\left\{p_{n}\right\}$ such that $p_{n}(\varphi(z)) \rightarrow z$ almost everywhere on $C$, from which it obviously follows that $\phi$ is univalent almost everywhere.

Let $D$ be the open unit disk, and for $\varphi$ in $H^{\infty}$ let $\varphi_{D}$ be the Poisson integral of $\varphi$. Thus $\varphi_{D}$ is a bounded analytic function in $D$ whose radial limits agree with $\varphi$ almost everywhere on $C$.

Proposition 3. If $\varphi$ is a generator of $H^{\infty}$ then $\varphi_{D}$ is univalent.

Proof. If $a$ is a point of $D$ then the evaluation functional $\varphi \rightarrow \varphi_{D}(a)$ on $H^{\infty}$ is weak-star continuous because it is induced by a function in $L^{1}$ (namely by the Poisson kernel for $\alpha$ ). The proposition is now immediate. A different proof can be based on the fact that for each $a$ in $D$, the function $h_{a}$ (defined in Section 3) is an eigenvector of $\varphi(U)^{*}$ with

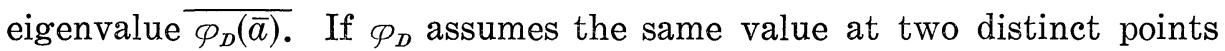
$a$ and $b$ of $D$, then the one dimensional subspace spanned by $h_{\bar{a}}+h_{\bar{b}}$ is invariant under $\varphi(U)^{*}$, although this subspace is not invariant under $U^{*}$.

\section{REFERENCES}

1. A. Beurling, On two problems concerning linear transformations in Hilbert space, Acta Math. 81 (1949), 239-255.

2. A. Brown and P. R. Halmos, Algebraic properties of Toeplitz operators, J. Reine Angew. Math. 213 (1963), 89-102.

3. J. Dixmier, Les Algèbres d'Opérateurs dans l'Espace Hilbertien, Gauthier-Villars, Paris, 1957.

4. B. Fuglede, A commutativity theorem for normal operators, Proc. Nat. Acad. Sci. 36 (1950). 35-40. 
5. R. W. Goodman, Prediction theory for unitary operators, Notices Amer. Math. Soc. 11 (1964), 221.

6. P. R. Halmos, Introduction to Hilbert Space and the Theory of Spectral Multiplicity, Chelsea, New York, 1957.

7. P. R. Halmos, Shifts on Hilbert spaces, J. Reine Angew. Math. 208 (1961), 102-112.

8. H. Helson and D. Lowdenslager, Invariant subspaces, Proceedings of the International Symposium on Linear Spaces, Jerusalem Academic Press, 1961, 251-265.

9. K. Hoffman, Banach Spaces of Analytic Functions, Prentice-Hall, Inc., Englewood Cliffs, N. J. 1962.

10. M. A. Naimark, Rings of operators in Hilbert space, Uspehi Mat. Nauk (N. S.) 4 (1949), no. 4 (32), 83-147. (in Russian)

11. A. I. Plesner and V. A. Rohlin, Spectral theory of linear operators, II, Uspehi Mat. Nauk (N. S.) 1 (1946), no.1, 71-191. (in Russian)

12. D. Sarason, On spectral sets having connected complement, to appear.

13. B. Sz.-Nagy, Spektraldarstellung Linearer Transformationen des Hilbertschen Raumes, Springer-Verlag, Berlin, 1942.

14. J. Wermer, On invariant subspaces of normal operators, Proc. Amer. Math. Soc. 3 (1952), 270-277.

UNIVERSity oF CALIFORNIA, BERKELEy 



\title{
PACIFIC JOURNAL OF MATHEMATICS
}

\author{
EDITORS
}

\section{H. SAMELSON}

Stanford University

Stanford, California

R. M. Blumenthal

University of Washington

Seattle, Washington 98105

\section{*J. DugundJI}

University of Southern California Los Angeles, California 90007

\section{RichaRd ARENS}

University of California

Los Angeles, California 90024

\section{ASSOCIATE EDITORS}
E. F. BECKENBACH
B. H. NEUManN
F. WOLF
K. YOSIDA

\section{SUPPORTING INSTITUTIONS}

\author{
UNIVERSITY OF BRITISH COLUMBIA \\ CALIFORNIA INSTITUTE OF TECHNOLOGY \\ UNIVERSITY OF CALIFORNIA \\ MONTANA STATE UNIVERSITY \\ UNIVERSITY OF NEVADA \\ NEW MEXICO STATE UNIVERSITY \\ OREGON STATE UNIVERSITY \\ UNIVERSITY OF OREGON \\ OSAKA UNIVERSITY \\ UNIVERSITY OF SOUTHERN CALIFORNIA
}

\author{
STANFORD UNIVERSITY \\ UNIVERSITY OF TOKYO \\ UNIVERSITY OF UTAH \\ WASHINGTON STATE UNIVERSITY \\ UNIVERSITY OF WASHINGTON \\ AMERICAN MATHEMATICAL SOCIETY \\ CHEVRON RESEARCH CORPORATION \\ TRW SYSTEMS \\ NAVAL ORDNANCE TEST STATION
}

\footnotetext{
Mathematical papers intended for publication in the Pacific Journal of Mathematics should be typewritten (double spaced). The first paragraph or two must be capable of being used separately as a synopsis of the entire paper. It should not contain references to the bibliography. Manuscripts may be sent to any one of the four editors. All other communications to the editors should be addressed to the managing editor, Richard Arens at the University of California, Los Angeles, California 90024 .

50 reprints per author of each article are furnished free of charge; additional copies may be obtained at cost in multiples of 50 .
}

The Pacific Journal of Mathematics is published monthly. Effective with Volume 16 the price per volume (3 numbers) is $\$ 8.00$; single issues, $\$ 3.00$. Special price for current issues to individual faculty members of supporting institutions and to individual members of the American Mathematical Society: $\$ 4.00$ per volume; single issues $\$ 1.50$. Back numbers are available.

Subscriptions, orders for back numbers, and changes of address should be sent to Pacific Journal of Mathematics, 103 Highland Boulevard, Berkeley 8, California.

Printed at Kokusai Bunken Insatsusha (International Academic Printing Co., Ltd.), No. 6, 2-chome, Fujimi-cho, Chiyoda-ku, Tokyo, Japan.

PUBLISHED BY PACIFIC JOURNAL OF MATHEMATICS, A NON-PROFIT CORPORATION

The Supporting Institutions listed above contribute to the cost of publication of this Journal, but they are not owners or publishers and have no responsibility for its content or policies.

* Paul A. White, Acting Editor until J. Dugundji returns. 


\section{Pacific Journal of Mathematics}

\section{Vol. 17, No. $3 \quad$ March, 1966}

Tsuyoshi Andô, Contractive projections in $L_{p}$ spaces ............... 391

Robert F. Brown, On a homotopy converse to the Lefschetz fixed point theorem ............................................... 407

Richard Albert Cleveland and Sandra Cleveland, On the multiplicative extension property................................ 413

Harold H. Johnson, An algebraic approach to exterior differential systems..................................... 423

Alan Cecil Lazer, The behavior of solutions of the differential equation

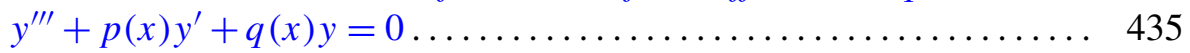

Judy Parr, Cohomology of cyclic groups of prime square order ......... 467

Donald Steven Passman, Groups whose irreducible representations have

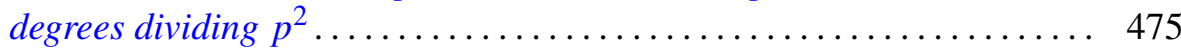

Ralph Tyrrell Rockafellar, Characterization of the subdifferentials of convex functions ........................................... 497

Donald Erik Sarason, Invariant subspaces and unstarred operator algebras...................................... 511

Donald Erik Sarason, Weak-star generators of $H^{\infty} \ldots \ldots \ldots \ldots \ldots \ldots . \ldots . \ldots$

Boris M. Schein, Homomorphisms and subdirect decompositions of semi-groups

Daniel Francis Shea, Jr., Functions analytic in a finite disk and having asymptotically prescribed characteristic

Zvi Ziegler, Generalized convexity cones 